\title{
Medulloepithelioma of the Ciliary Body Revealing by a Leukocoria
}

\author{
Sara Idmane, Mehdi Khamaily, Loubna El Maaloum, Bouchra Allali, and Asmaa El Kettani
}

\begin{abstract}
Leukocoria is a cause of consultation in pediatric ophthalmology, congenital catarct as well as retinoblastoma constitute the most frequent causes, nevertheless, a rarer cause can be at the origin of this white glow of the pupil: the Medullo epithelium of the ciliary body.

We report the case of a 5-year-old girl in whom a medullo epithelium of the ciliary body was suspected, revealed by a simple leukocoria, confirmed histologically after enucleation.
\end{abstract}

Index Terms - non pigmented ciliary epithelium, Leukocoria, enucleation, embryonal tumor.

\section{INTRODUCTION}

Leukocoria is a frequent cause of consultation in pediatric ophthalmology, congenital catarct as well as retinoblastoma constitute the most frequent causes, nevertheless, a rarer cause can be at the origin of this white glow of the pupil: the Medullo epithelium of the ciliary body.

\section{CASE REPORT}

We report the case of a little girl of 5 years old, who consults in pediatric ophthalmology for a leukocoria in the right eye observed by the parents.

The ophthalmological examination showed a visual acuity of the left eye at $4 / 20$ with no target refraction. The patient had no strabismus or nystagmus.

Anterior segment: a clear cornea with an anterior chamber slightly reduced in depth in the nasal side.

The examination under sedation after dilation: intraocular pressure at $10 \mathrm{mmhg}$, nasal lens opacity obstructing the visual axis, (Fig. 1, 2).

The Skepens ophthalmoscope showed a normal retina.

The mode B ocular ultrasound objectified a mass of the ciliary body in the nasal slightly driving the iris forward with a nasal lens opacification, without retinal lesion (Fig. 3).

The UBM showed multiple cysts of the nasal ciliary body with zonular rupture (Fig. 4).

The ocular scan objectified the mass of the ciliary body without retinal lesion or suspect calcification. (Fig. 5).

Taking into account the age of the patient, the clinical,

Published on August 23, 2020.

Sara Idmane, University hospital center Ibn Rochd, Hassan II University, Morocco.

Mehdi Khamaily, Mohammed V University Rabat, Morocco.

(corresponding e-mail: mehdi.khamaily@gmail.com)

Loubna El Maaloum, University hospital center Ibn Rochd, Hassan II University, Morocco.

Bouchra Allali, University hospital center Ibn Rochd, Hassan II University, Morocco.

Asmaa El Kettani, University hospital center Ibn Rochd, Hassan II University, Morocco. echographic and CT aspects, the most likely diagnosis was the medullo epithelium of the ciliary body.

An extension report was requested which turned out to be negative.

After explanation of the risks, the possible therapeutic alternatives as well as the visual prognosis or even vital, and after a parental consent, we opted for an enucleation for diagnostic and therapeutic purposes.

The anatomo pathological examination objectified a teratoid medullo epithelium.

The patient's follow-up over 1 year did not show any local recurrence.

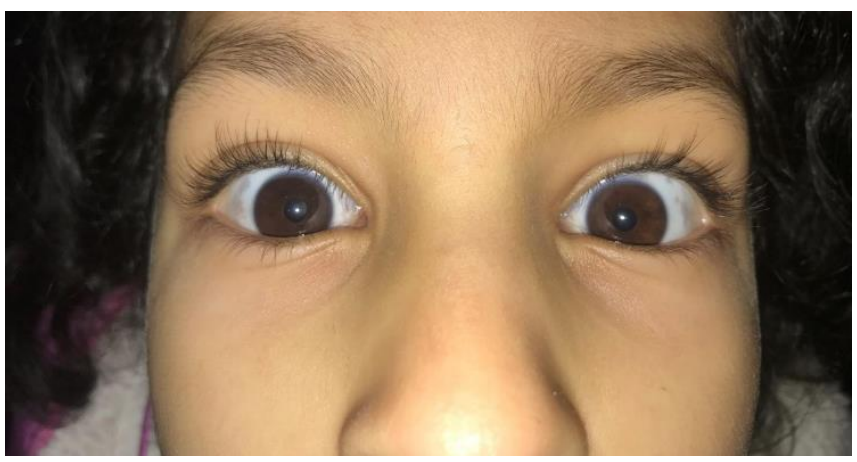

Fig. 1. Leukocoria of the right eye.

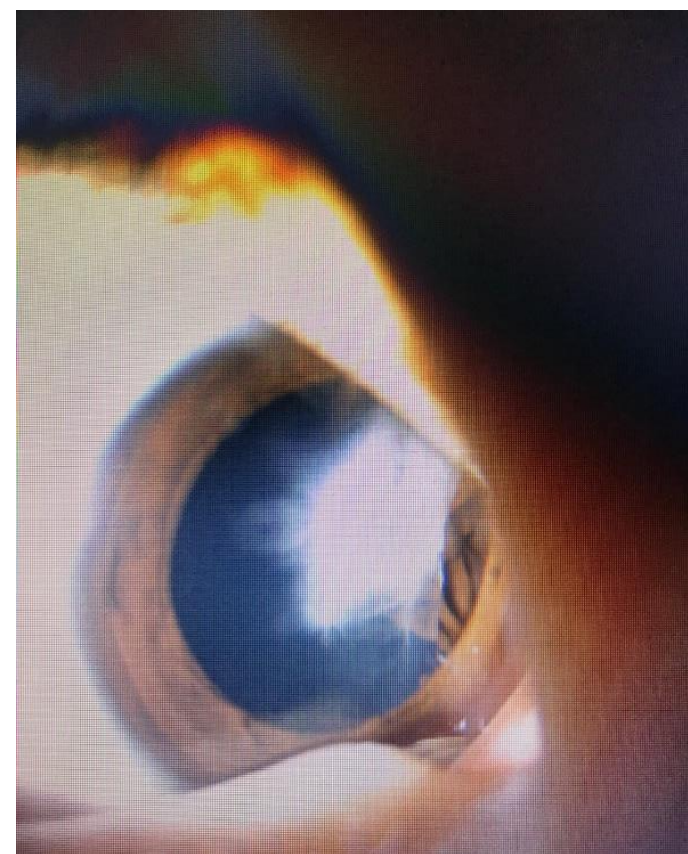

Fig. 2. After dilation, mass of the ciliary body nasally with clouding of the lens. 


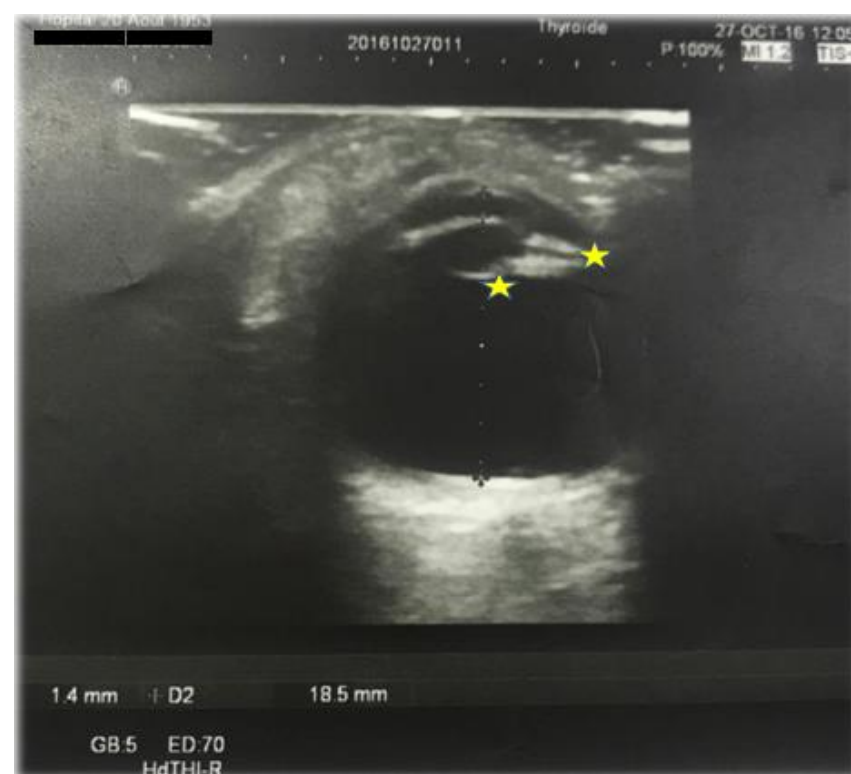

Fig. 3. Mode B ultrasound showing the mass of the cilary body with clouding of the lens (yellow stars) without suspect retinal mass.
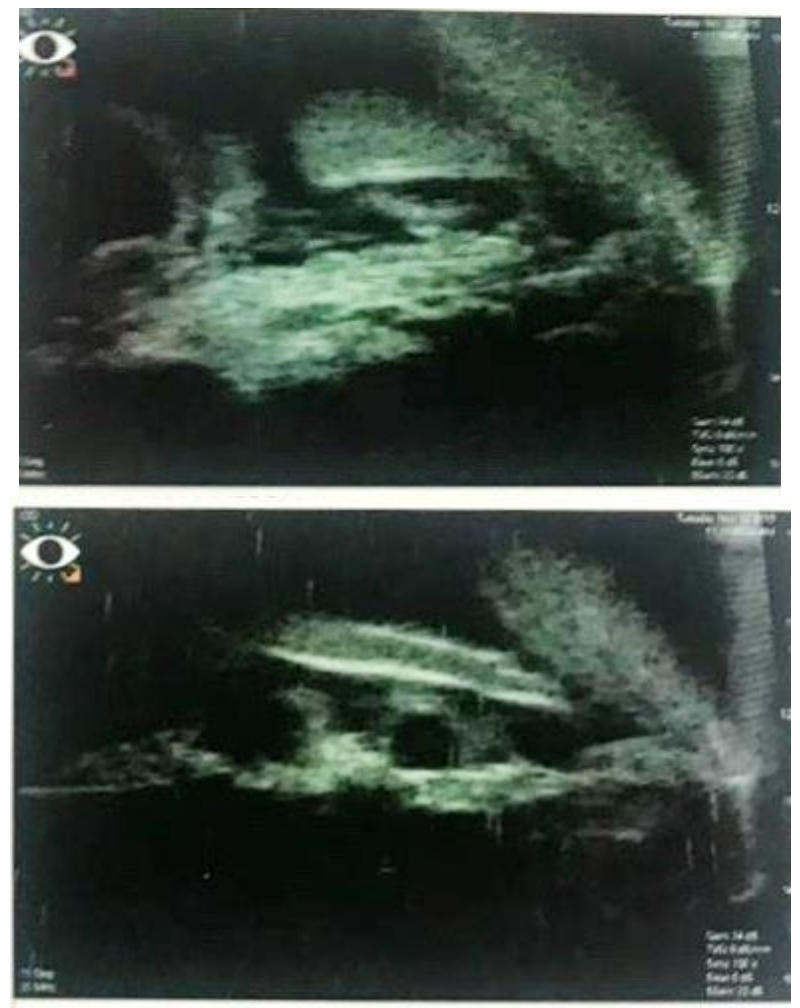

Fig. 4. Multiple cysts of the nasal ciliary body with zonular rupture.

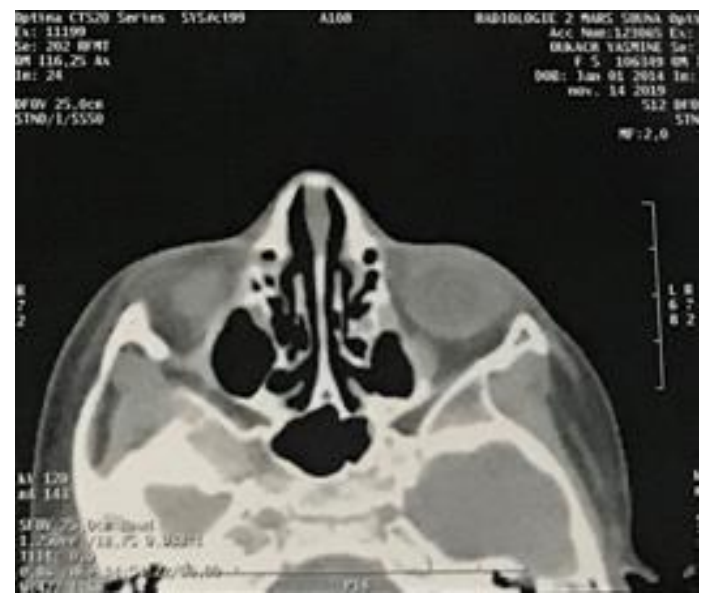

Fig. 5. Eye scanner showing no retinal calcifications.

\section{DISCUSSION}

After several nomenclature of this tumor of the ciliary body since 1904, Grinken named it: medulloepithelium in 1931[1].

It is a malignant tumor of origin of the clear epithelium of the ciliary body since the internal layer of the optical cup, rarer than retinoblastoma [2].

The average age at the time of diagnosis is 5 years, the main complication of which is the neovascularization of the iris and the retro lental cyclitic membrane in $21.5 \%$, the clouding of the lens in $20 \%$ followed by secondary glaucoma in $18.5 \%$ [3].

The malignancy of this tumor is estimated at $80 \%$, the histological aspect is often banded or tubular neuroepithelial formations, sometimes tumor cysts can detach and float in the CA. [4]

Zimmerman proposed a classification, subsequently adopted by Reese and then by the WHO (1971), nonteratoid medullo-epitheliomas: made up of cells from a single germinal embryonic layer, teratoid medulloepitheliomas made up of cells made from two different layers of germinal embryonic tissue which may contain bone, cartilage or nerve elements [5].

The treatment is enucleation in the majority of cases $(60$ to $70 \%$ depending on the series. Sometimes tumor excision: partial lamellar sclero-uvectomy or iridocyclectomy, small tumor limited to 3 or 4 hourly quadrants. (23\%) [6].

Recently, brachytherapy using an iodine-125 disc or ruthenium-106 plate, either as an additional treatment (localized tumor post-excision) or as a primary treatment for the tumor [7]-[8].

The often poor visual prognosis with a metastatic spread rate is only $8 \%$ [3], furthermore 4 deaths have been described in a series of 56 cases from Broughton and Zimmerman [9].

\section{CONCLUSION}

The medulloepithelium remains a very rare tumor, of which it is necessary to be wary of atypical signs in particular leucocorie, the iris rubella or the spontaneous hyphema in the small child.

The progress of surgery has greatly changed the visual prognosis, particularly with increasingly conservative treatments.

\section{REFERENCES}

[1] Grinker RR. "Gliomas of the retina including results of studies with silver impregnations". Arch Ophthalmol, 1931; 5: 920-35.

[2] John R Priest et al, "Ciliary body medulloepithelioma: four cases associated with pleuropulmonary blastomada report from the International Pleuropulmonary Blastoma Registry". Br J Ophthalmol 2011; 95:1001e1005. doi:10.1136/bjo.2010.189779.

[3] Swathi Kaliki et al. "Ciliary Body Medulloepithelioma Ophthalmology" 2013; 120:2552-2559 a 2013 by the American Academy of Ophthalmology.

[4] Sameeksha H Tadepalli et al. "Intraocular medulloepithelioma - A review of clinical features, DICER 1 mutation, and management". Indian journal ophthalmology, 2019 Jun; 67(6): 755-762. doi: 10.4103/ijo.IJO_845_19.

[5] Zimmerman LE. Verhoeff's "terato-neuroma". A critical reappraisal in light of new observations and current concepts of embryonic 
tumors. The Fourth Frederick H. Verhoeff Lecture. Am J Ophthalmol 1971; 72:1039-57.

[6] Shields JA, Eagle RC Jr, Shields CL, De Potter P. “Acquired neoplasms of the nonpigmented ciliary epithelium (adenoma and adenocarcinoma)". Ophthalmology, 1996; 103:2007-16.

[7] Cassoux N, Charlotte F, Sastre X, et al. Conservative surgical treatment of medulloepithelioma of the ciliary body [letter]. Arch Ophthalmol 2010; 128:380-1.

[8] Poon DS, et al. "Ruthenium-106 plaque brachytherapy in the primary management of ocular medulloepithelioma". Ophthalmology, 2015; 122: 1949-51.

[9] Broughton WL et Zimmerman LE et al. "A clinicopathologic of 56 cases ofintra ocular medulloepitheliomas", Am J Ophthalmol, 1978; 85: 407-418. 